\title{
CARACTERIZACIÓN DE LAS HABILIDADES DEL PENSAMIENTO CRÍTICO PARA EL DESARROLLO DEL CONOCIMIENTO DIDÁCTICO DEL CONTENIDO EN PROFESORES DE CIENCIAS NATURALES
}

\author{
Characterization of Critical Thinking Skills \\ for the development of didactic content knowledge \\ in Natural Science Teachers
}

\author{
Marcelo Augusto SALICA \\ Universidad Nacional del Comahue. \\ Facultad de Ciencias de la Educación Argentina \\ Correo-e: marcelo_salica@face.uncoma.edu.ar
}

Recibido: 12.12.2017; Aceptado: 11.3.2018; Publicado: 30.06.2018

Ref. Bibl. M. A. SALICA. Caracterización de las habilidades del pensamiento crítico para el desarrollo del Conocimiento Didáctico del Contenido en profesores de ciencias naturales. Enseñanza E Teaching, 36, 1-2018, 199-221.

RESUMEN: El estudio del Conocimiento Didáctico del Contenido (CDC) constituye un paradigma de investigación relevante en el contexto internacional e iberoamericano. Este tema de trascendencia actual se ha transformado en un marco conceptual de metanivel como herramienta fundamental para la mejora de la calidad de la formación del profesorado de ciencias. Aunque la diversidad de estrategias metodológicas utilizadas para la documentación del CDC se focaliza en la caracterización de este conocimiento, las metodologías ignoran cuáles son las habilidades cognitivas para su desarrollo. El objetivo de la presente investigación reside en contrastar el desarrollo de las habilidades del pensamiento crítico por medio de una intervención didáctica fundamentada en la naturaleza de la ciencia y evaluar los efectos y disposiciones pretest y postest, en docentes $(n=29)$ de escuela secundaria que enseñan Fisicoquímica o Biología. Los resultados indican una mejora a nivel global 
MARCELO AUGUSTO SALICA

CARACTERIZACIÓN DE LAS HABILIDADES DEL PENSAMIENTO CRÍTICO PARA EL DESARROLLO DEL CONOCIMIENTO DIDÁCTICO DEL CONTENIDO EN PROFESORES DE CIENCIAS NATURALES

en 4 de 5 habilidades y con marcadas diferencias en las habilidades del pensamiento crítico entre: los/las profesores que enseñan Biología y Fisicoquímica, y los docentes con formación pedagógica-didáctica y formación técnico-disciplinar. Esta diferencia deriva en consecuencias para el desarrollo de competencias transversales para una enseñanza fundamentada en la Naturaleza de la Ciencia y la Tecnología (NdCyT). Desde la perspectiva docente, la naturaleza mixta del test se perfila como una herramienta rigurosa para identificar y caracterizar las habilidades del pensamiento crítico por contraste de hipótesis para el desarrollo del CDC caracterizando el perfil profesional y disciplinar.

Palabras clave: pensamiento crítico; conocimiento didáctico del contenido; formación docente; ciencias naturales.

SUMMARY: The study of Didactic Content Knowledge (PCK) constitutes a relevant research paradigm in the international and Ibero-American context. Its productivity in this issue of current importance has been transformed into a meta-level conceptual framework as a fundamental tool to improve the quality of science teacher training. Although the diversity of methodological strategies used for their documentation focuses on the characterization of this content pedagogical knowledge, they ignore what the cognitive skills for their development are. The objective of this research is to contrast the development of critical thinking skills through a didactic intervention based on the nature of science and evaluate the effects and pretest and posttest dispositions, in teachers $(n=29)$ of high school who teach Physicochemistry or Biology. The results indicate a global improvement in 4 out of 5 skills and differences in critical thinking skills among the professors who teach Biology and Physicochemistry, and the teachers with pedagogical-didactic training and technical-disciplinary training. This difference results in consequences for the development of transversal competences for a teaching based on the nature of science and technology. From a teaching perspective, the mixed nature of the test is emerging as a rigorous tool to identify and characterize critical thinking by contrast of hipothesis skills for the development of didactic content knowledge according to the professional and disciplinary profile.

Key words: critical thinking; pedagogical content knowledge; teacher training; natural sciences.

\section{INTRODUCCIÓN}

La enseñanza de las ciencias en el siglo xxi demanda que los estudiantes logren competencia científica, y para alcanzar esta meta es necesario que el profesorado no solo conozca la ciencia de su campo disciplinar que pretende enseñar, sino que desarrolle su competencia didáctica para hacerlo. A raíz de lo anterior, el complejo escenario que se le presenta a la comunidad de educadores en una sociedad de cambios acelerados y de necesidad de modernización de la educación 
CARACTERIZACIÓN DE LAS HABILIDADES DEL PENSAMIENTO CRÍTICO PARA EL DESARROLLO DEL CONOCIMIENTO DIDÁCTICO DEL CONTENIDO EN PROFESORES DE CIENCIAS NATURALES

no siempre resulta amigable para lo científico y tecnológico. En este contexto, uno de los paradigmas que adquiere gran relevancia a nivel internacional, dado el creciente número de publicaciones para el desarrollo del profesorado, se denomina "Conocimiento Didáctico del Contenido (CDC)» y la "Naturaleza de la Ciencia y la Tecnología (NdCут)», estos son 2 de los 13 tópicos que Garritz (2010) denomina "paradigmas de la enseñanza de las ciencias» (p. 316). El concepto "Pedagogical Content Knowledge» (РСK) es introducido por Shulman en 1986, hoy traducido al español como Conocimiento Didáctico del Contenido (CDC) (Blanco, Mellado y Ruiz, 1995) o Conocimiento Pedagógico del Contenido (CDC) (Garritz y Trinidad-Velasco, 2004; Garritz, 2013).

Los expertos como Garritz, Daza-Rosales y Lorenzo (2014) y en contraste con la revisión bibliográfica que aportan Verdugo-Perona, Solaz-Portolés y Sanjosé-López (2017), dan cuenta de que el CDC es un modelo extremadamente útil de razonamiento y acción pedagógica. En concreto, estos autores permiten conocer qué es y qué no es el CDC y qué se ha investigado sobre este paradigma. Las investigaciones se focalizan mayormente en cómo y qué características tiene el CDC, cómo se ha determinado en el profesorado, su relación con el conocimiento disciplinar a enseñar y con el aprendizaje de los estudiantes, su utilización en la formación del profesorado y en qué temas científicos se ha estudiado. Sin embargo, se ha prestado poca o escasa atención en conocer las habilidades del pensamiento o los recursos cognitivos que se requieren para ser un buen docente; dado que las investigaciones para caracterizar el CDC se realizan con docentes que se identifican como "profesores/as excelentes». Esto último lleva a cuestionar cómo se define un buen profesional de ciencias y cuáles son sus habilidades cognitivas de razonamiento que se presupone que los hace ser excelentes en la enseñanza. En el contexto donde se realiza esta indagación, conviven dos tipos de profesionales que enseñan ciencias en la escuela secundaria: a) profesores con formación pedagógico-didáctica y b) profesionales con formación técnico-disciplinar. Este segundo grupo son caracterizados como idóneos o habilitantes para el ejercicio de la docencia, constituidos por profesionales de campos académicos como técnicos, médicos, licenciados, farmacéuticos, ingenieros, entre otros.

Por otro lado, especialistas como Farré y Lorenzo (2009) reconocen que el estudio del CDC es complejo de ser documentado, ya que una de las estrategias metodológicas utilizadas para su documentación con mayor éxito consiste en el análisis del discurso utilizando un cuestionario semiestructurado propuesto por Loughran, Berry y Mulhall (2012), denominado Representaciones del Contenido (ReCo) y adaptado al contexto iberoamericano por Garritz y Trinidad-Velasco (2004), Padilla, Ponce de León, Rembado y Garritz (2008). Sin embargo, el conocimiento profesional específico que desarrollan los profesores ofrece una oportunidad para conocer el nivel de desempeño de las habilidades del pensamiento crítico (PC) que manifiestan y las configuraciones que este conocimiento puede orientar en la construcción de un CDC específico. 
CARACTERIZACIÓN DE LAS HABILIDADES DEL PENSAMIENTO CRÍTICO PARA EL DESARROLLO DEL CONOCIMIENTO DIDÁCTICO DEL CONTENIDO EN PROFESORES DE CIENCIAS NATURALES

Así planteada esta cuestión, se advierte que la complejidad del escenario descrito y el reto que el mismo exige a los educadores hacen que la formación del profesorado en la competencia transversal ligada a la competencia científica sea tan importante como el desarrollo de las habilidades del PC. Esto exige asumir la siguiente premisa: «saber ciencias no equivale a tener competencia transversal para enseñar ciencias». A su vez, contrastar las habilidades del PC entre cada grupo de profesionales que enseña ciencias permitiría identificar, transferir y retroalimentar, es decir, transcontextualizar las cualidades de los conocimientos y su razonamiento didáctico para la educación científica de los estudiantes.

Para afrontar este reto, el objetivo de la presente investigación reside en caracterizar las habilidades del pensamiento crítico por medio de una intervención didáctica fundamentada en la Ndcyт y evaluar los efectos y disposiciones pretest y postest, en docentes de escuela secundaria que enseñan ciencias naturales (Fisicoquímica y Biología). Este objetivo permitirá identificar las habilidades del PC en los profesionales de la educación científica, a partir de las destrezas y los rasgos epistémicos que conforman el PC desde sus propias concepciones o pensamientos espontáneos. Caracterizar el CDC a partir del desarrollo de la competencia transversal, por medio de un análisis cuantitativo, representa un estudio imprescindible que permitirá explicar y comprender mejor esta capacidad humana indispensable de carácter transversal y de especial relevancia para la enseñanza de la ciencia y la tecnología.

\subsection{La competencia transversal, el pensamiento crítico (PC)}

El reto profesional que se le presenta al profesorado es de gran magnitud. Desarrollar las competencias científicas conlleva no solo conocer sobre el contenido disciplinar, sino desarrollar habilidades y destrezas propias de las ciencias para el logro de competencias transversales para la formación de los profesionales de la educación científica. Esto implica asumir por parte del profesorado que «saber ciencias no equivale a tener competencia transversal como el pensamiento crítico ligadas al pensamiento científico para enseñar ciencias». A partir de ello, se aborda la definición de competencia científica, como uno de los conceptos que fundamenta el presente estudio y que resulta de una profunda discusión de expertos como Pedrinaci, Caamaño, Cañal y de Pro, concebida como:

[...] un conjunto integrado de capacidades para utilizar el conocimiento científico a fin de describir, explicar y predecir fenómenos naturales; para comprender los rasgos característicos de la ciencia; para formular e investigar problemas e hipótesis; así como para documentarse, argumentar y tomar decisiones personales y sociales sobre el mundo natural y los cambios que la actividad humana genera en él (2012: 31).

Aunque la idea de competencia presente una contradictoria ambigüedad y en consecuencia no esté exenta de controversias, en el presente estudio se aborda 
CARACTERIZACIÓN DE LAS HABILIDADES DEL PENSAMIENTO CRÍTICO PARA EL DESARROLLO

DEL CONOCIMIENTO DIDÁCTICO DEL CONTENIDO EN PROFESORES DE CIENCIAS NATURALES

la idea de competencia transversal que la Organización para la Cooperación y el Desarrollo Económico (OCDE, 2005, 2010) y la UNESCO (2005) señalan como necesaria para el desarrollo en la sociedad del siglo xxI, fundamentada en el desarrollo del pensamiento crítico. Las habilidades del PC constituyen un conjunto de habilidades que de acuerdo a varios autores como Halpern (1998), Facione (1990) y López (2012) llevan una carga cognitiva que exige del uso de estructuras mentales de orden superior como razonar, argumentar, reconocer pruebas, validar conocimiento, elaborar hipótesis, tomar decisiones y resolver problemas, las cuales guardan relación con las habilidades propias del pensamiento científico. A partir de esta evidente afinidad entre pensamiento crítico y pensamiento científico para la enseñanza de las ciencias naturales, resulta necesario considerar las exigencias que conlleva el desarrollo de la competencia científica como un componente de la competencia transversal. El solapamiento conceptual, dado a través de la idea de la transversalidad y en acuerdo con Martín-Barbero (2003), no resulta de una mera cuestión de disciplinas e interdisciplinas. Involucra la transferencia transcontextual de las habilidades del PC (Halpern, 1998). Para alcanzar este propósito, los educadores deberán desarrollar sus competencias específicas didáctico-pedagógicas concebidas a partir del desarrollo del CDC. Al respecto Clark (citado en Coronado, 2013) se refiere a «lo que tienen en común las actividades específicas del profesorado, como es la manipulación del conocimiento, entendida como una combinación muy variada de esfuerzos tendientes al descubrimiento, la conservación, la depuración, la transmisión y la aplicación del mismo» (p. 101).

\subsection{El desarrollo del pensamiento crítico, como indicador del Conocimiento Didáctico del Contenido}

De acuerdo con las disertaciones anteriores, las competencias van más allá de un simple uso de saberes y destrezas limitadas al conocimiento disciplinar. Alcanzar su desarrollo en el profesorado conlleva un doble esfuerzo de parte de los/las profesores/as. Este esfuerzo demanda comprender y hacer comprender un tema particular del campo disciplinar a partir de las características del contexto sociocultural en el que se desempeña el profesorado en ciencias como ciudadanos responsables. Para llevar adelante este desafío el profesorado debe realizar un doble ejercicio: ser ciudadanos y educadores científicamente competentes, lo cual exige destrezas que no son obvias. Este doble ejercicio incluye la comprensión de lo que facilita o dificulta el aprendizaje de ese contenido específico, así como las concepciones y conocimientos previos de los estudiantes sobre el tema. El conjunto de conocimientos que desarrolla el profesorado en ejercicio es lo que se denomina Conocimiento Didáctico del Contenido (CDC), definido por Shulman (1987) como «la amalgama especial de contenido y pedagogía que constituye el dominio único de los profesores, su propia y especial forma de comprensión profesional» (p. 6). Así, la misma noción del CDC exige innegablemente el desarrollo de la competencia transversal antes definida, lo que proporciona criterios para seleccionar, evaluar y 
CARACTERIZACIÓN DE LAS HABILIDADES DEL PENSAMIENTO CRÍTICO PARA EL DESARROLLO DEL CONOCIMIENTO DIDÁCTICO DEL CONTENIDO EN PROFESORES DE CIENCIAS NATURALES

tomar decisiones basadas en las propias "creencias o concepciones espontáneas" (Álvarez-Suárez, Cepero-Espinosa, Arce y Perales-Palacios, 2013) sobre las ciencias vinculada a la práctica educativa. Es por ello que resulta de gran importancia promover el pensamiento científico basado en el desarrollo y caracterización de las habilidades del pensamiento crítico. Puesto que toda actividad de pensamiento se sitúa en un conjunto de conocimientos o creencias epistemológicas que lo fundamentan, el CDC resulta de la interacción de los diferentes componentes que se organizan en cuatro categorías de conocimiento: disciplinar, psicopedagógica, contextual y metadisciplinar. De acuerdo con Penagos y Lozano (2014), estas categorías interactúan de forma independiente, pero mutuamente implicadas, lo que constituye un marco teórico de metanivel, es decir, un constructo complejo para mejorar la formación de los profesionales de la educación.

Llegar a tener una comprensión amplia sobre el CDC resulta de gran importancia para consolidar la caracterización de las habilidades del pensamiento crítico y para lograr formular una definición. Caracterizar las habilidades del pensamiento crítico admite de manera operativa y concreta conocer diferentes grados de dominio de estas habilidades en el profesorado en ejercicio, en función de las disciplinas que se imparten y de la formación académica que poseen y que resultan prioritarias para su formación. A partir de esta caracterización, el nivel de logro se manifiesta en la práctica, promovida por los contextos de desempeño del profesorado. Para alcanzar una mejor comprensión, Molina-Patlan, Morales-Martínez y Valenzuela-González (2016) sostienen que, cuando una persona alcanza una comprensión amplia sobre su conocimiento y su saber, entonces se llega a un conocimiento epistemológico más asertivo. De esta manera, resulta de mayor interés y utilidad conocer no tanto lo que el docente sabe, cree o siente, sino saber analizar y caracterizar cómo utiliza sus bases cognitivas para enfrentar distintas situaciones didácticas demandadas por contenidos específicos en contextos variados. Desde la perspectiva del estudio descripto, caracterizar las habilidades del PC que desarrollan los docentes invita a la realización del presente estudio desde un enfoque metodológico más explícito, nuevo y riguroso. Esto posibilita conocer los problemas epistemológicos particulares que remiten a las didácticas específicas, particularmente en la Biología y la Fisicoquímica, y desde el contexto de desempeño del grupo de docentes participantes.

\subsection{La evaluación del pensamiento crítico a partir de la naturaleza de la ciencia y la tecnología}

En este estudio, la idea de pensamiento crítico es entendida, de acuerdo con Paul y Elder (2003), como un constructo complejo de «orden superior» que, en coherencia con Halpern (1998), está formado por un conjunto de 5 habilidades cognitivas de alto nivel: comprobación de hipótesis, razonamiento verbal, análisis de argumentos, probabilidad e incertidumbre, toma de decisiones y resolución de problemas. Muchos son los autores que coinciden en que el pensamiento crítico es 
CARACTERIZACIÓN DE LAS HABILIDADES DEL PENSAMIENTO CRÍTICO PARA EL DESARROLLO DEL CONOCIMIENTO DIDÁCTICO DEL CONTENIDO EN PROFESORES DE CIENCIAS NATURALES

el pensamiento «razonado y reflexivo» (Dewey, 1998; Ennis, 1993, 1996) sobre qué hacer o en qué creer donde se incluyen procesos de "regulación y metacognición" (Facione, 1990; Lipman, 1988) cuya transversalidad, como antes se argumentó, permite su relación en múltiples áreas.

La intervención didáctica integra aspectos de la Naturaleza de la Ciencia y la Tecnología (Ndсут) desde un enfoque Ciencia, Tecnología y Sociedad (CTS) que, de acuerdo con Acevedo-Díaz y García-Carmona (2016), su enseñanza en la formación del profesorado está íntimamente relacionada y sugieren el desarrollo del CDC para una implementación eficaz. El dominio de la Naturaleza de la Ciencia es un "Conocimiento sobre cómo se construye la ciencia, los métodos que se usan para validar las teorías o sustituirlas por otras, el papel de la comunidad científica en todo ello y las relaciones CTS» (Pedrinaci et al., 2012: 278; Abd-el-Khalick y Akerson, 2009). Este enfoque propone un modelo para la enseñanza de las habilidades del pensamiento científico que permite su transferencia a través de instrumentos de evaluación e intervención didáctica que contienen andamiajes didácticos para mejorar el desarrollo del PC para la educación científica. La caracterización de las habilidades del PC conlleva identificar las habilidades a partir de la facultad mental en la cual el profesorado de ciencias en ejercicio reflexiona, cuestiona, examina y hace uso eficaz de sus recursos cognitivos (Kuhn, 1999). Por otro lado, Nieto y Saiz (2011) comprenden que han surgido nuevos factores indispensables para llegar a un pensamiento crítico. Estos factores son la comprensión epistemológica del pensamiento crítico y de las creencias sobre el pensamiento. En consecuencia, cuando se habla de pensamiento en la formación docente, resulta necesario identificar las cualidades de este pensamiento, muy estudiado en el campo de la didáctica de las ciencias. Una de ellas es el denominado "pensamiento espontáneo o creencias del profesorado" (Mellado, 1999; Imbernón, 2010). El pensamiento espontáneo, de acuerdo con Álvarez-Suárez (2013: 349), está vinculado al conocimiento que tienen sobre las ciencias, sus decisiones, limitaciones, competencias y actitudes ante su profesión que repercute y condiciona su planificación e intervención didáctica, lo que puede resultar contradictorio. Este tipo de pensamiento o creencias espontáneas no implica en el profesorado, necesariamente, el desarrollo espontáneo del pensamiento formal, basado en estructuras de conjuntos lógicos. Su desarrollo, según Pozo y Carretero (1987), requiere de su instrucción mediante estrategias o esquemas para la solución de problemas que no se adquieren unitariamente, ya que dependen decisivamente del contenido de la tarea.

Para finalizar, el desarrollo y la evaluación de las habilidades del pensamiento crítico como competencia transversal ligada al pensamiento científico resulta en una condición necesaria con influencia directa en los cuatro componentes del CDC del profesorado en ejercicio. Como se ha fundamentado previamente, su evaluación presenta desafíos metodológicos por ser un constructo complejo y de orden superior, al evaluar habilidades cognitivas de nivel superior y de orden inferior, que ponen en acción las creencias o concepciones espontáneas del profesorado. Es por ello que el test de Halpern (1998) presenta características superadoras que 
CARACTERIZACIÓN DE LAS HABILIDADES DEL PENSAMIENTO CRÍTICO PARA EL DESARROLLO DEL CONOCIMIENTO DIDÁCTICO DEL CONTENIDO EN PROFESORES DE CIENCIAS NATURALES

lo hacen diferente a otras metodologías que existen actualmente. Esta metodología utiliza situaciones cotidianas o escenarios similares a las encontradas en la vida real, como situaciones sociales complejas, que exigen aplicar habilidades transversales. De acuerdo a Nieto, Saiz y Orgaz (2009), el análisis de las propiedades psicométricas hace que el instrumento de evaluación resulte representativo de lo que puede encontrarse en un periódico o en una discusión cotidiana. Otra característica es que utiliza un doble formato de pregunta, que lo hace diferente a otras pruebas de pensamiento crítico. Este formato mixto combina respuesta abierta/ construida y preguntas de elección/decisión forzada. Su valor reside en que resultan apropiadas para evaluar capacidades cognitivas separables, como efecto de la intervención didáctica en los participantes. Esto exige en el profesorado tener que buscar conscientemente y seleccionar los conocimientos y habilidades apropiadas, fundamentadas en sus creencias o concepciones y pensamientos espontáneos, lo que exige poner en interacción los componentes del CDC.

\section{Metodología}

\subsection{Muestra y contexto}

La investigación se realizó durante el desarrollo del programa de fortalecimiento en la enseñanza de las ciencias naturales (Fisicoquímica y Biología). Este programa es una línea de capacitación de la Dirección Nacional de Gestión Curricular y Formación Docente del Consejo Federal de Educación con el Consejo Provincial de Educación de Neuquén. El dispositivo consistió en encuentros jurisdiccionales por módulos de dos días de $8 \mathrm{~h}$ reloj cada uno y un encuentro de discusión de cierre de $8 \mathrm{~h}$, realizado 60 días después y mediado por un aula virtual. La muestra de investigación se define como «fortuita de diseño longitudinal de cohorte» según Hernández, Fernández y Baptista (2006), constituida por 58 docentes en servicio, procedentes de 27 escuelas secundarias estatales distribuidas en la zona norte, centro y sur de la provincia.

Las principales características de la muestra figuran resumidas en la Tabla 1, categorizadas en dos grupos definidos del siguiente modo: a) docentes profesores (DP) son aquellos que poseen el título de profesores provenientes de carreras de grado del profesorado con formación pedagógica y didáctica, b) docentes habilitantes (DH), conformado por profesionales de grado académico con título técnico/ disciplinar como en ingeniería, medicina, licenciaturas y farmacia, entre otros. De la muestra total, finalizaron con el programa de formación docente 29 (50\%) participantes constituyendo el grupo experimental en el 2016, quienes recibieron el tratamiento didáctico interdisciplinar y aplicación del test de PC. 
MARCELO AUGUSTO SALICA

CARACTERIZACIÓN DE LAS HABILIDADES DEL PENSAMIENTO CRÍTICO PARA EL DESARROLLO DEL CONOCIMIENTO DIDÁCTICO DEL CONTENIDO EN PROFESORES DE CIENCIAS NATURALES

TABLA 1

Caracterización de los participantes de la muestra

\begin{tabular}{|c|c|c|}
\hline \multirow[t]{2}{*}{ CATEgORÍA } & \multicolumn{2}{|c|}{$\begin{array}{c}\text { GRUPO } \\
\text { EXPERIMENTAL }\end{array}$} \\
\hline & FA & \% VÁLIDO \\
\hline Docentes Profesores (DP) & 14 & 48,27 \\
\hline Docentes Habilitantes (DH) & 15 & 51,72 \\
\hline Edad promedio (años) & \multicolumn{2}{|c|}{39,85} \\
\hline \multirow{2}{*}{ Género } & Hombre & Mujer \\
\hline & 5 & 24 \\
\hline \multirow[t]{2}{*}{ Asignatura } & $\begin{array}{l}\text { Biología } \\
\text { (B) }\end{array}$ & $\begin{array}{l}\text { Fisicoquímica } \\
\text { (FQ) }\end{array}$ \\
\hline & 10 & 19 \\
\hline Total & \multicolumn{2}{|c|}{29} \\
\hline
\end{tabular}

FUENTE: Elaboración propia.

\subsection{Procedimiento de la intervención}

La Tabla 2 expone el diseño de la metodología de la investigación con la aplicación el test de PC. El contenido de los módulos 3 y 4 aborda temas referidos a: los problemas científicos disciplinares con relevancia social y los problemas sociales complejos.

TABLA 2

Diseño fortuito pretest y postest

\begin{tabular}{|c|c|c|c|}
\hline GRUPO & $\begin{array}{c}\text { PRETEST } \\
\text { EVALUACIÓN }\end{array}$ & $\begin{array}{c}\text { INTERVENCIÓN } \\
\text { DIDÁCTICA } \\
\text { INTERDISCIPLINAR }\end{array}$ & $\begin{array}{c}\text { POSTEST } \\
\text { EVALUACIÓN }\end{array}$ \\
\hline Experimental & PC & Ejes/módulos \\
Tiempo & $x / 07 / 2016$ & y 4 & $30 / 09 / 2016$ \\
\hline
\end{tabular}

FUENTE: Elaboración propia 


\subsection{Instrumentos}

Los instrumentos de investigación que se aplican en este proyecto se agrupan en dos clases: instrumentos de evaluación de la mejora e instrumentos de intervención didáctica.

\subsection{Instrumento de evaluación}

Las cuestiones aplicadas del test de PC presentan las siguientes características: utilizan situaciones cotidianas y similares a las encontradas en la vida real y poseen un doble formato de pregunta. Se seleccionaron 5 situaciones o escenarios, cada uno correspondiente a una de las cinco habilidades definidas por el test, de un total de 25 situaciones diferentes. En cada situación se plantea un problema sobre el que se formula una pregunta abierta y tras ello, se pide que elijan una alternativa que resuelva la situación o cuestión planteada. De acuerdo a Halpern, este doble formato de preguntas permite conocer, por un lado, si el que responde el test manifiesta un uso espontáneo de la habilidad y, por otro, si es capaz de usarla cuando se le señala que es necesaria, aun cuando no haya reconocido que fuera necesaria.

Procedimiento: los participantes completaron el test en formato papel y lápiz y el máximo de tiempo de realización fue de 90 minutos, en el pretest y postest. El pretest se realizó antes de iniciar las actividades de intervención didáctica y el postest se efectuó 60 días después de la intervención didáctica. Los participantes desconocían la metodología de trabajo pre-/pos-test. Para ello, se adoptó el modelo de evaluación que propone Diane Halpern (2006), test HCTAEs (Halpern Critical Thinking Assesment Using Everyday Situations). Las habilidades del pensamiento crítico contempladas para su evaluación están divididas en las siguientes cinco categorías cognitivas:

a) Razonamiento verbal (Rv): incluye habilidades necesarias para comprender y defenderse de las técnicas persuasivas presentes en el lenguaje cotidiano.

b) Uso de la probabilidad y la incertidumbre ( $\mathrm{Pi}$ ): dado que muy pocos eventos en la vida pueden ser conocidos con certeza, el uso correcto de probabilidades acumulativas, exclusivas y contingentes debe jugar un rol crítico en casi cualquier decisión.

c) Elaboración de hipótesis (Hi): esta categoría implica que los sujetos actúen como científicos intuitivos para predecir y explicar/conjeturar eventos.

d) Análisis de argumento (Aa): un argumento es un conjunto de enunciados con, al menos, una conclusión, una razón que la sustenta y un contraargumento. En la vida real, los argumentos son complejos, con razones contrarias a la conclusión, con supuestos establecidos y no establecidos, con información irrelevante y con etapas intermedias entre las conclusiones y la evidencia que las sustenta. 
CARACTERIZACIÓN DE LAS HABILIDADES DEL PENSAMIENTO CRÍTICO PARA EL DESARROLLO DEL CONOCIMIENTO DIDÁCTICO DEL CONTENIDO EN PROFESORES DE CIENCIAS NATURALES

e) Toma de decisiones y resolución de problemas (Rp): las habilidades consideradas en las categorías anteriores se relacionan con la toma de decisiones y la resolución de problemas. Involucran en esta categoría la generación y selección de alternativas y su evaluación, lo cual implica también un solapamiento con el pensamiento creativo, relacionado con la generación de alternativas y la reformulación de problemas y metas.

El test de PC ofrece rasgos importantes que lo diferencian de los demás instrumentos de evaluación dado que resulta innovador y reduce las limitaciones metodológicas al ofrecer dos tipos de respuestas, a) una parte de respuesta construida y b) una parte con ítems de respuesta rápida o decisión forzada (por ejemplo, opción múltiple, clasificación o calificación de alternativas). La naturaleza multimodal del instrumento posibilita realizar un análisis cualitativo y cuantitativo sobre las respuestas de los sujetos participantes, mejorando la interpretación de los datos. Esto se debe a que los psicólogos cognitivos diferencian entre memoria libre y procesos de reconocimiento en memoria y estos dos tipos de preguntas están diseñados para aprovechar los diferentes procesos cognitivos. Cada habilidad tiene un peso diferente sobre el total de las 5 habilidades, que se detallan en la siguiente Tabla 3, donde se explicitan los escenarios evaluados por cada habilidad.

TABLA 3

Escenarios de cada habilidad evaluada y porcentaje de ponderación por categoría en cuanto a su importancia relativa

\begin{tabular}{|l|l|}
\hline \multicolumn{1}{|c|}{$\begin{array}{c}\text { HABILIDAD } \\
\text { (\% PESO) }\end{array}$} & \multicolumn{1}{c|}{ SITUACIÓN/ESCENARIO } \\
\hline $\begin{array}{l}\text { Razonamiento } \\
\text { verbal (11\%) }\end{array}$ & $\begin{array}{l}\text { El director de la Escuela Primaria Los Álamos envió esta dura nota } \\
\text { a los profesores de dibujo de la escuela: «En mis recientes visitas } \\
\text { a las clases de dibujo, he observado que algunos profesores } \\
\text { permiten a los alumnos que dibujen lo que quieran. Esto, por } \\
\text { definición, no es enseñar. Si los alumnos en sus clases de Dibujo } \\
\text { pintan los mismos cuadros que pintarían en su casa o por su } \\
\text { cuenta, el profesor no aporta nada al desarrollo del alumno y } \\
\text { por tanto, no está enseñando». }\end{array}$ \\
\hline $\begin{array}{l}\text { Probabilidad e } \\
\text { incertidumbre (13\%) }\end{array}$ & $\begin{array}{l}\text { Andrés encontró hace poco un artículo en el periódico que } \\
\text { muestra el incremento en el tamaño de la población mundial y } \\
\text { en la producción total de alimentos. Según este artículo, en la } \\
\text { proporción que vamos, los alimentos se agotarán dentro de 80 } \\
\text { años aproximadamente. }\end{array}$ \\
\hline
\end{tabular}


CARACTERIZACIÓN DE LAS HABILIDADES DEL PENSAMIENTO CRÍTICO PARA EL DESARROLLO DEL CONOCIMIENTO DIDÁCTICO DEL CONTENIDO EN PROFESORES DE CIENCIAS NATURALES

\begin{tabular}{|l|l|}
\hline \multicolumn{1}{|c|}{$\begin{array}{c}\text { HABILIDAD } \\
\text { (\% PESO) }\end{array}$} & \multicolumn{1}{c|}{ SITUACIÓN/ESCENARIO } \\
\hline $\begin{array}{l}\text { Elaboración de } \\
\text { hipótesis (22\%) }\end{array}$ & $\begin{array}{l}\text { Un informe reciente aparecido en una revista para padres y } \\
\text { profesores que muestra que los adolescentes que fuman suelen } \\
\text { obtener peores calificaciones en clase. A medida que aumenta } \\
\text { el número de cigarrillos por día, disminuye el promedio de } \\
\text { las calificaciones. Una sugerencia que hace el informe es que } \\
\text { podríamos mejorar el rendimiento escolar evitando el consumo } \\
\text { de tabaco entre los adolescentes. }\end{array}$ \\
\hline $\begin{array}{l}\text { Análisis de } \\
\text { argumento (23\%) }\end{array}$ & $\begin{array}{l}\text { Algunas universidades están pensando en añadir un nuevo } \\
\text { requisito para licenciarse (titularse): que cada estudiante preste } \\
\text { algún servicio público de utilidad para poder graduarse. }\end{array}$ \\
\hline $\begin{array}{l}\text { Toma de decisión } \\
\text { y resolución de } \\
\text { problema (31\%) }\end{array}$ & $\begin{array}{l}\text { Te han contratado para mejorar la productividad y el nivel } \\
\text { general de satisfacción con el trabajo en una cadena de montaje } \\
\text { de automóviles sin aumentar los costos. Descubres que el } \\
\text { entusiasmo de los trabajadores ha sido muy bajo por cerca } \\
\text { de un año y parece seguir así en este momento. Hay una ola de } \\
\text { calor que está afectando al trabajo de todos y hace que los } \\
\text { empleados vayan más despacio rompiendo el ritmo de la cadena } \\
\text { de montaje. }\end{array}$ \\
\hline
\end{tabular}

FUENTE: Elaboración propia.

\subsection{Instrumento de intervención didáctica}

Los instrumentos de intervención didáctica son la planificación y diseño de secuencias de enseñanza y aprendizaje (SEA) sobre un rasgo específico de la Ndcyt. La SEA contiene el plan articulado de actividades de aprendizaje orientadas a las concepciones de los participantes. Las pautas sobre reacciones esperadas del profesorado se fundamentan en la investigación, adaptadas al nivel de formación, a las características y contenidos del dominio Ndcyt, los supuestos epistemológicos, las perspectivas de aprendizaje, los enfoques pedagógicos actuales y las características del contexto educativo. La estructura didáctica adoptada para la SEA se basa en el "ciclo de aprendizaje 7E» de Eisenkraft (2003), citado en Vázquez y Rodríguez (2013), que propone un esquema de siete fases: Extraer, Enganchar, Explorar, Explicar, Elaborar, Extender y Evaluar. Durante la intervención didáctica, se presenta el tema con la técnica denominada role playing. Para ello, las personas participantes forman grupos de tres integrantes simulando un equipo científico interdisciplinario. Cada participante asume el rol de científico/a en una de las tres disciplinas: física, química o biología. El trabajo consiste en la indagación del fenómeno, exploración y descripción desde una perspectiva interdisciplinaria. 
MARCELO AUGUSTO SALICA

CARACTERIZACIÓN DE LAS HABILIDADES DEL PENSAMIENTO CRÍTICO PARA EL DESARROLLO

DEL CONOCIMIENTO DIDÁCTICO DEL CONTENIDO EN PROFESORES DE CIENCIAS NATURALES

\section{Resultados}

Como se describe en la metodología, el formato de respuesta mixta del test admite conocer el desempeño de las habilidades del PC. Estos dos tipos de preguntas/respuestas están diseñados para aprovechar los diferentes procesos cognitivos, lo que permite identificar el tipo de razonamiento asociado a estructuras de tipo lógico-disciplinar y didáctico. El efecto de la intervención didáctica se valora al comparar los resultados entre las puntuaciones pre- y postest por cada habilidad. El análisis de datos se realizó con el programa informático SPSs $^{\circledR}$. Se efectuaron pruebas de significación "p-value", a partir de las siguientes hipótesis definidas para el contraste de resultados: I) existen diferencias significativas en las habilidades del PC entre los grupos de docentes participantes (Docentes Profesores «DP» y Docentes Habilitantes «DH») en función del tipo de formación académica; II) existen diferencias significativas entre la prueba pre-/postest por cada habilidad y entre la puntuación total de las 5 habilidades de pensamiento crítico. La prueba "d" de Cohen determina el tamaño del efecto sobre el valor "r" de Spearman. Con las dos pruebas anteriores se establece la correlación y cálculo del porcentaje de éxito para contrastar las diferentes habilidades en función del tipo de respuesta doble: construida y/o forzada. El índice «d» de Cohen es independiente del tamaño de la muestra, en función de ello el criterio aplicado para medir el tamaño del efecto se considera relevante cuando es mayor que $0,30(d>0,30)$. El signo que se obtiene junto al índice del tamaño del efecto determina la dirección de la mejora entre los dos grupos de estudio. El signo negativo determina la mejora del efecto hacia el grupo de los docentes habilitantes (DH) y, en caso contrario, el efecto de mejora se orienta hacia el grupo de los docentes profesores (DP).

\subsection{Parámetros globales del grupo}

La prueba estadística aplicada para evaluar cada una de las habilidades permite conocer cuáles fueron significativamente afectadas en función de la intervención didáctica y la dirección de la mejora relativa al grupo de comparación. Los resultados del ANOva para la prueba de diferencia inter-grupos, docentes profesores (DP) y docentes habilitantes (DH) se resume en la siguiente Tabla 4. Estos resultados expresan la puntuación total por habilidad, igualmente ponderada entre la respuesta construida y las de elección forzada. 
MARCELO AUGUSTO SALICA

CARACTERIZACIÓN DE LAS HABILIDADES DEL PENSAMIENTO CRÍTICO PARA EL DESARROLLO DEL CONOCIMIENTO DIDÁCTICO DEL CONTENIDO EN PROFESORES DE CIENCIAS NATURALES

TABLA 4

Contraste de resultados inter-grupo por habilidad de PC

\begin{tabular}{|c|c|c|c|}
\hline \multirow{2}{*}{$\begin{array}{c}\text { HABILIDADES } \\
\text { DEL PC }\end{array}$} & GRUPO & Prueba estadística & GRUPO \\
\hline & $\begin{array}{c}\text { DH } \\
(-)\end{array}$ & $P$-VALUE $\mathrm{Y}$ « $D$ » DE COHEN & $\begin{array}{l}\text { DP } \\
(+)\end{array}$ \\
\hline Razonamiento verbal «Rv» & & $\mathrm{p}=0,86 ; \mathrm{d}=0,07$ & $*$ \\
\hline Probabilidad e incertidumbre «Pi» & & $p=0,36 ; d=0,39$ & $*$ \\
\hline Análisis de argumento "Aa" & $*$ & $p=0,23 ; d=-0,45$ & \\
\hline Hipótesis «Hi» & $*$ & $\mathrm{p}=0,06 ; \mathrm{d}=-0,77$ & \\
\hline Resolución de problemas (Rp) & $*$ & $\mathrm{p}=, 000 ; \mathrm{d}=-2,82$ & \\
\hline
\end{tabular}

EL uso del asterisco (*) destaca el grupo que tuvo mejoras con el tratamiento didáctico dado por el resultado de la prueba "d» de Cohen. FuENTE: Elaboración propia.

En los datos de la Tabla 4 se observan las habilidades que presentan mejoras y diferencias estadísticamente significativas. El grupo DP tuvo mejoras en las habilidades de razonamiento verbal, probabilidad e incertidumbre y análisis de argumentos, sin diferencias estadísticamente significativas. El grupo DH presenta mejoras del efecto en las habilidades de análisis de argumento, elaboración de hipótesis y resolución de problemas, con diferencia estadísticamente significativa en esta última.

En la Tabla 5 se muestran los resultados de las pruebas para cada tipo de respuesta de las cinco habilidades evaluadas.

TABLA 5

Resultados de los estadísticos del tipo de respuesta por habilidad del PC

\begin{tabular}{|c|c|c|c|c|c|c|c|}
\hline \multicolumn{8}{|c|}{ PRUEBAS ESTADÍSTICAS } \\
\hline $\begin{array}{l}\text { HABILIDADES } \\
\text { (\% PESO) }\end{array}$ & $\begin{array}{c}\text { TIPO DE } \\
\text { PREGUNTA/ } \\
\text { RESPUESTA }\end{array}$ & MEDIA & $\begin{array}{l}\text { DESV. } \\
\text { TIP. }\end{array}$ & $\begin{array}{l}\text { «D» DE } \\
\text { COHEN }\end{array}$ & $\begin{array}{c}\text { «R» DE } \\
\text { SPEARMAN }\end{array}$ & $\begin{array}{c}\% \\
\text { ÉXITO }\end{array}$ & P-VALOR \\
\hline \multirow{2}{*}{$\begin{array}{l}\text { Razonamiento } \\
\text { verbal } \\
\text { «Rv» }(11 \%)\end{array}$} & $\begin{array}{l}\text { Respuesta } \\
\text { construida }\end{array}$ & $-0,234$ & 0,632 & $-0,11$ & 0,45 & 44,64 &, 386 \\
\hline & $\begin{array}{l}\text { Decisión } \\
\text { forzada }\end{array}$ & 0,322 & 0,097 & 0,14 & 0,57 & 56,94 &, 264 \\
\hline \multirow{2}{*}{$\begin{array}{l}\text { Probabilidad e } \\
\text { incertidumbre } \\
\text { «Pi (13\%) }\end{array}$} & $\begin{array}{l}\text { Respuesta } \\
\text { construida }\end{array}$ & 0,643 & 0,708 & 0,26 & 0,63 & 63,24 & ,014 \\
\hline & $\begin{array}{l}\text { Decisión } \\
\text { forzada }\end{array}$ & $-0,432$ & 0,177 & $-0,20$ & 0,40 & 39,98 &, 128 \\
\hline
\end{tabular}


MARCELO AUGUSTO SALICA

CARACTERIZACIÓN DE LAS HABILIDADES DEL PENSAMIENTO CRÍTICO PARA EL DESARROLLO DEL CONOCIMIENTO DIDÁCTICO DEL CONTENIDO EN PROFESORES DE CIENCIAS NATURALES

\begin{tabular}{|c|c|c|c|c|c|c|c|}
\hline \multicolumn{8}{|c|}{ Pruebas estadísticas } \\
\hline $\begin{array}{l}\text { HABILIDADES } \\
\text { (\% PESO) }\end{array}$ & $\begin{array}{c}\text { TIPO DE } \\
\text { PREGUNTA/ } \\
\text { RESPUESTA }\end{array}$ & MEDIA & $\begin{array}{l}\text { DESV. } \\
\text { TIP. }\end{array}$ & $\begin{array}{l}\text { «D» DE } \\
\text { COHEN }\end{array}$ & $\begin{array}{c}\text { "R» DE } \\
\text { SPEARMAN }\end{array}$ & $\begin{array}{c}\% \\
\text { ÉXITO }\end{array}$ & P-VALOR \\
\hline \multirow{2}{*}{$\begin{array}{l}\text { Análisis de } \\
\text { argumento } \\
\text { "Aa" (23\%) }\end{array}$} & $\begin{array}{l}\text { Respuesta } \\
\text { construida }\end{array}$ & 0,163 & 0,543 & 0,07 & 0,54 & 53,59 & , 498 \\
\hline & $\begin{array}{l}\text { Decisión } \\
\text { forzada }\end{array}$ & 0,596 & 0,422 & 0,25 & 0,62 & 62,36 &, 053 \\
\hline \multirow{2}{*}{$\begin{array}{l}\text { Hipótesis } \\
\text { «Hi» }(22 \%)\end{array}$} & $\begin{array}{l}\text { Respuesta } \\
\text { construida }\end{array}$ & 0,542 & 0,667 & 0,23 & 0,61 & 61,34 &, 074 \\
\hline & $\begin{array}{l}\text { Decisión } \\
\text { forzada }\end{array}$ & 0,415 & 0,139 & 0,18 & 0,59 & 58,85 &, 056 \\
\hline \multirow{2}{*}{$\begin{array}{l}\text { Resolución } \\
\text { de problemas } \\
\text { «p» (31\%) }\end{array}$} & $\begin{array}{l}\text { Respuesta } \\
\text { construida }\end{array}$ & 0,987 & 0,561 & 0,38 & 0,69 & 69,10 &, 001 \\
\hline & $\begin{array}{l}\text { Decisión } \\
\text { forzada }\end{array}$ & 0,140 & 0,229 & 0,06 & 0,53 & 53,11 &, 597 \\
\hline \multicolumn{2}{|c|}{$\begin{array}{l}\text { Promedio de las cinco } \\
\text { Habilidades }\end{array}$} & $-0,112$ & 0,182 & 0,99 & 0,44 & 61,09 & 0,03 \\
\hline
\end{tabular}

FUENTE: Elaboración propia.

Se observa que dos respuestas construidas y dos respuestas de decisión forzada son estadísticamente significativas/afectadas como efecto de mejora. El promedio global de las cinco habilidades de pensamiento crítico evaluadas (pre- y postest) presenta diferencias estadísticamente significativas con un nivel de confianza del 95\% ( $\mathrm{p}=0,03<0,05)$. De acuerdo a la $\mathrm{r}$ de Spearman 8 de las 10 respuestas ( 5 respuestas construidas y 5 respuestas forzadas) obtienen un promedio ponderado con un porcentaje de éxito del 50\%, lo que se considera relevante. Para la muestra global, el valor $\mathrm{d}=0,99$, es decir, que la efectividad o efecto de la intervención didáctica es alta, con un 61,09\% de éxito como resultado de la intervención didáctica.

La Figura 1 representa la mejora global con base en la puntuación total igualmente ponderada entre la respuesta construida y las preguntas de elección forzada para las 5 habilidades del total de la muestra de participantes $(n=29)$. 
FiguRA 1

Índice global de las habilidades del pensamiento crítico

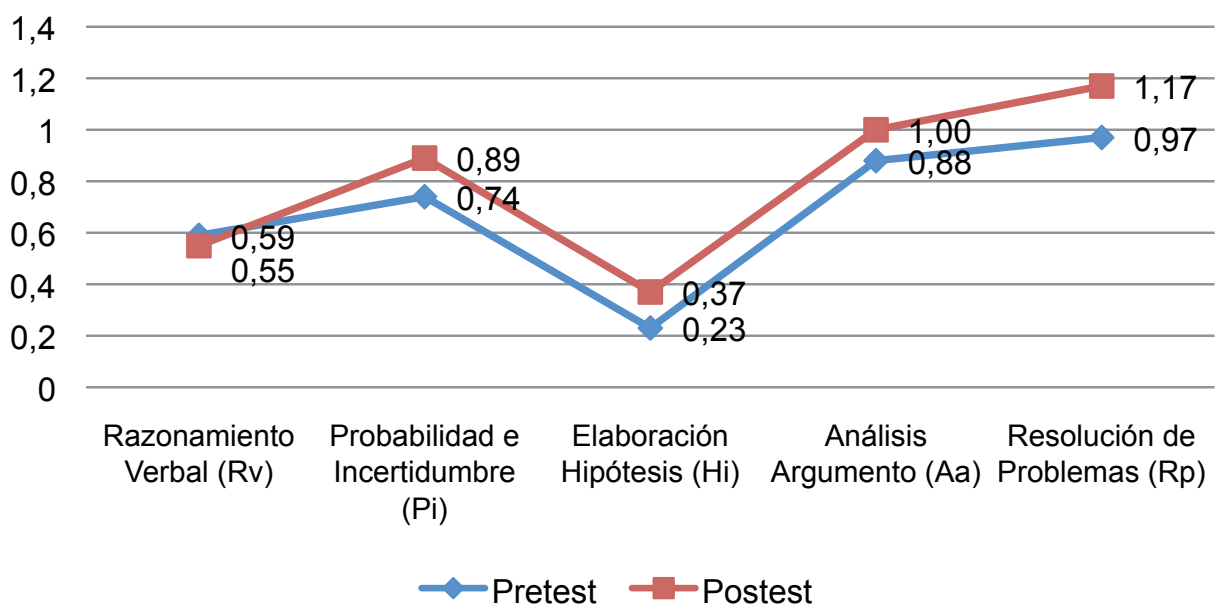

FUENTE: Elaboración propia.

\subsection{Comparaciones y diferencias entre grupos por asignaturas}

En la Tabla 6 se exponen los resultados obtenidos en cada una de las cinco habilidades del pensamiento crítico discriminado por disciplinas (docentes que enseñan biología y docentes que enseñan físico-química). 
MARCELO AUGUSTO SALICA

CARACTERIZACIÓN DE LAS HABILIDADES DEL PENSAMIENTO CRÍTICO PARA EL DESARROLLO DEL CONOCIMIENTO DIDÁCTICO DEL CONTENIDO EN PROFESORES DE CIENCIAS NATURALES

\section{TABLA 6}

Contraste de los estadísticos por categorías del tipo de respuesta, aplicada a cada grupo en función de la disciplina

\begin{tabular}{|c|c|c|c|c|c|}
\hline \multirow[b]{2}{*}{$\begin{array}{l}\text { HABILIDADES } \\
\text { DEL PC (TEST } \\
\text { DE HALPERN) }\end{array}$} & \multirow[b]{2}{*}{$\begin{array}{c}\text { TIPO DE } \\
\text { RESPUESTA }\end{array}$} & \multicolumn{4}{|c|}{ (INCLUYEN DOCENTES GRUPO A Y B) } \\
\hline & & $\begin{array}{l}\text { BIOLOGÍA } \\
\text { (PRE-/POS-) }\end{array}$ & $\begin{array}{c}\text { PROMEDIO } \\
\text { DEL } \\
\text { P-VALOR } \\
\text { DE LOS } \\
\text { TIPOS DE } \\
\text { RESPUESTA }\end{array}$ & $\begin{array}{l}\text { FISICOQUÍMICA } \\
\text { (PRE-/POS-) }\end{array}$ & $\begin{array}{c}\text { PROMEDIO } \\
\text { DEL P-VALOR } \\
\text { DE LOS TIPOS } \\
\text { DE RESPUESTA }\end{array}$ \\
\hline \multirow{2}{*}{$\begin{array}{c}\text { Razonamiento } \\
\text { verbal (Rv) }\end{array}$} & $\begin{array}{l}\text { Respuesta } \\
\text { construida }\end{array}$ & 1,000 & \multirow{2}{*}{0,914} & 0,346 & \multirow{2}{*}{0,460} \\
\hline & $\begin{array}{l}\text { Decisión } \\
\text { forzada }\end{array}$ & 0,317 & & 0,414 & \\
\hline \multirow{2}{*}{$\begin{array}{l}\text { Probabilidad } \\
\text { incertidumbre } \\
\qquad(\mathrm{Pi})\end{array}$} & $\begin{array}{l}\text { Respuesta } \\
\text { construida }\end{array}$ & 0,015 & \multirow{2}{*}{0,012} & 0,199 & \multirow{2}{*}{0,484} \\
\hline & $\begin{array}{l}\text { Decisión } \\
\text { forzada }\end{array}$ & 0,916 & & 0,048 & \\
\hline \multirow{2}{*}{ Hipótesis (Hi) } & $\begin{array}{l}\text { Respuesta } \\
\text { construida }\end{array}$ & 0,366 & \multirow{2}{*}{0,380} & 0,111 & \multirow{2}{*}{0,074} \\
\hline & $\begin{array}{l}\text { Decisión } \\
\text { forzada }\end{array}$ & 1,000 & & 0,034 & \\
\hline \multirow{2}{*}{$\begin{array}{l}\text { Análisis de } \\
\text { argumento } \\
\text { (Aa) }\end{array}$} & $\begin{array}{l}\text { Respuesta } \\
\text { construida }\end{array}$ & 0,705 & \multirow{2}{*}{0,173} & 0,380 & \multirow{2}{*}{0,393} \\
\hline & $\begin{array}{l}\text { Decisión } \\
\text { forzada }\end{array}$ & 0,078 & & 0,415 & \\
\hline \multirow{2}{*}{$\begin{array}{c}\text { Resolución } \\
\text { de problemas } \\
\text { (Rp) }\end{array}$} & $\begin{array}{l}\text { Respuesta } \\
\text { construida }\end{array}$ & 0,066 & \multirow{2}{*}{0,240} & 0,014 & \multirow{2}{*}{0,002} \\
\hline & $\begin{array}{l}\text { Decisión } \\
\text { forzada }\end{array}$ & 0,157 & & 0,035 & \\
\hline
\end{tabular}

FUENTE: Elaboración propia.

Estos resultados permiten identificar las diferencias en el nivel de desempeño de las 5 habilidades en los participantes que enseñan biología, obteniendo diferencia estadísticamente significativa en una sola habilidad, vinculada con la probabilidad e incertidumbre. Para el caso de los participantes que enseñan físico-química, desarrollaron sus habilidades del pensamiento crítico en tres de las cinco habilidades evaluadas, con cambios estadísticamente significativos. 
MARCELO AUGUSTO SALICA

CARACTERIZACIÓN DE LAS HABILIDADES DEL PENSAMIENTO CRÍTICO PARA EL DESARROLLO

DEL CONOCIMIENTO DIDÁCTICO DEL CONTENIDO EN PROFESORES DE CIENCIAS NATURALES

\subsection{Comparaciones y diferencias entre grupos por formación académica}

Los resultados permiten discriminar las cinco habilidades en función a las características de los participantes ordenados en la siguiente Tabla 7.

\section{TABLA 7}

Contraste de los estadísticos por habilidad del PC aplicado a los grupos en función de la formación académica

\begin{tabular}{|c|c|c|c|c|c|}
\hline \multirow[b]{2}{*}{$\begin{array}{l}\text { HABILIDADES } \\
\text { DEL PC }\end{array}$} & \multirow[b]{2}{*}{$\begin{array}{c}\text { TIPO DE } \\
\text { RESPUESTA }\end{array}$} & \multicolumn{4}{|c|}{$\begin{array}{l}\text { CADA GRUPO DP O DH SE CONSTITUYE } \\
\text { POR LAS DOS ASIGNATURAS }\end{array}$} \\
\hline & & $\begin{array}{l}\text { GRUPO DH } \\
\text { (PRE-/POS-) }\end{array}$ & $\begin{array}{c}\text { PROMEDIO } \\
\text { DEL P-VALOR } \\
\text { DE LOS TIPOS } \\
\text { DE RESPUESTA }\end{array}$ & $\begin{array}{l}\text { GRUPO DP } \\
\text { (PRE-/POS-) }\end{array}$ & $\begin{array}{c}\text { PROMEDIO } \\
\text { DEL P-VALOR } \\
\text { DE LOS TIPOS } \\
\text { DE RESPUESTA }\end{array}$ \\
\hline \multirow{2}{*}{$\begin{array}{c}\text { Razonamiento } \\
\text { verbal (Rv) }\end{array}$} & $\begin{array}{l}\text { Respuesta } \\
\text { construida }\end{array}$ & 0,257 & \multirow{2}{*}{0,457} & 0,159 & \multirow{2}{*}{0,258} \\
\hline & $\begin{array}{l}\text { Decisión } \\
\text { forzada }\end{array}$ & 0,564 & & 0,046 & \\
\hline \multirow{2}{*}{$\begin{array}{l}\text { Probabilidad e } \\
\text { incertidumbre } \\
\text { (Pi) }\end{array}$} & $\begin{array}{l}\text { Respuesta } \\
\text { construida }\end{array}$ & 0,271 & \multirow{2}{*}{0,559} & 0,038 & \multirow{2}{*}{0,071} \\
\hline & $\begin{array}{l}\text { Decisión } \\
\text { forzada }\end{array}$ & 0,140 & & 0,368 & \\
\hline \multirow{2}{*}{ Hipótesis (Hi) } & $\begin{array}{l}\text { Respuesta } \\
\text { construida }\end{array}$ & 0,548 & \multirow{2}{*}{0,385} & 0,074 & \multirow{2}{*}{0,076} \\
\hline & $\begin{array}{l}\text { Decisión } \\
\text { forzada }\end{array}$ & 0,025 & & 0,655 & \\
\hline \multirow{2}{*}{$\begin{array}{c}\text { Análisis de } \\
\text { argumento } \\
\text { (Aa) }\end{array}$} & $\begin{array}{l}\text { Respuesta } \\
\text { construida }\end{array}$ & 1,000 & \multirow{2}{*}{0,284} & 0,431 & \multirow{2}{*}{0,286} \\
\hline & $\begin{array}{l}\text { Decisión } \\
\text { forzada }\end{array}$ & 0,093 & & 0,220 & \\
\hline \multirow{2}{*}{$\begin{array}{l}\text { Resolución } \\
\text { de Problemas } \\
\quad(\mathrm{Rp})\end{array}$} & $\begin{array}{l}\text { Respuesta } \\
\text { construida }\end{array}$ & 0,070 & \multirow{2}{*}{0,012} & 0,018 & \multirow{2}{*}{0,047} \\
\hline & $\begin{array}{l}\text { Decisión } \\
\text { forzada }\end{array}$ & 0,034 & & 0,473 & \\
\hline
\end{tabular}

FUENTE: Elaboración propia.

En los datos se encuentra que el grupo constituido por docentes profesores (DP) con formación psicopedagógico-didáctica específica alcanzaron cambios estadísticamente significativos en tres de las cinco habilidades, siendo estas habilidades: razonamiento verbal, probabilidad e incertidumbre y resolución de problemas, 
CARACTERIZACIÓN DE LAS HABILIDADES DEL PENSAMIENTO CRÍTICO PARA EL DESARROLLO

DEL CONOCIMIENTO DIDÁCTICO DEL CONTENIDO EN PROFESORES DE CIENCIAS NATURALES

sumando un 55\% del peso ponderado en importancia relativa al conjunto de las 5 habilidades (100\%) del PC. Mientras que el grupo conformado por docentes con formación técnico-científica (DH) alcanzaron cambios estadísticamente significativos en dos habilidades: la elaboración de hipótesis y la resolución de problemas, lo que constituye un 53\% del peso ponderado por categoría relativa al 100\% de las 5 habilidades del PC. Este contraste complementa el resultado obtenido por medio del ANOva de la Tabla 4. En el caso del grupo DP, los efectos de cambios estadísticamente significativos se dan en dos habilidades que requieren un uso espontáneo de la habilidad, lo que exige procesamiento cognitivo de orden superior y un efecto de mejora en la respuesta forzada. Para el caso del grupo DH, los cambios significativos obtenidos corresponden a dos respuestas de reconocimiento, cuyas respuestas requieren del procesamiento cognitivo de orden inferior.

\section{DisCUSIÓN}

El enfoque metodológico utilizado como nueva forma de evaluar y caracterizar el razonamiento específico del contenido a partir de las habilidades del pensamiento crítico muestra la potencia de esta propuesta metodológica. El contraste de los resultados permite, desde el punto de vista del diagnóstico, comparar y contrastar las diferencias en el nivel de desempeño de las habilidades del PC de cada grupo de participantes. A nivel global, la diferencia porcentual ponderada del intra-grupos sobre el total de las 5 habilidades del PC no resulta cuantitativamente significativa. Sin embargo, la característica del instrumento de evaluación de doble respuesta admite no solo cuantificar el nivel de desempeño, sino también realizar un análisis cualitativo del mismo. En este sentido, el análisis cualitativo exhibe una marcada diferencia en el tipo de habilidades de cada grupo cuando utilizan sus recursos cognitivos para enfrentar situaciones didácticas fundamentadas en la NdcyT. De lo anterior, se encuentra que el grupo DP hace uso de procesos cognitivos de orden superior e inferior en tres habilidades. Particularmente, el grupo de docentes que enseña fisicoquímica exhibe el uso de varias habilidades de orden superior, lo que permite identificar un conocimiento didáctico específico que puede vincularse a una mejor comprensión sobre las metaciencias. Ciertamente, este tipo de conocimiento revela la disposición del pensamiento y la epistemología del profesorado sobre la enseñanza, que resulta de la interacción de diversas habilidades cuando el docente genera alternativas para resolver situaciones disímiles. Ello deriva en el desarrollo de una competencia transversal más apropiada. Por su parte, el grupo de docentes que enseña biología presenta una disposición menos adecuada respecto del grupo anterior de acuerdo con los resultados obtenidos. En términos generales, las carencias encontradas podrían atribuirse a una formación en contenidos metacientíficos precarios o insuficientes, lo que puede derivar en un tratamiento inadecuado de los contenidos de la educación científica en el aula.

Respecto de los docentes habilitantes (DH), se encuentra que el grupo carece de habilidades que se puedan valorar como adecuadas para enfrentar situaciones 
CARACTERIZACIÓN DE LAS HABILIDADES DEL PENSAMIENTO CRÍTICO PARA EL DESARROLLO DEL CONOCIMIENTO DIDÁCTICO DEL CONTENIDO EN PROFESORES DE CIENCIAS NATURALES

didácticas fundamentadas en la NdcyT. Esto se debe a que el conocimiento metacientífico exige del uso de las destrezas del PC como competencia transversal. Si bien su nivel de desempeño exhibe un alto porcentaje en dos habilidades significativas como "hipótesis» y "toma de decisiones y resolución de problemas», las mismas se reconocen mediante la activación de procesos cognitivos inferiores. Esta disposición exhibe un pensamiento que implica utilizar la memoria de reconocimiento, lo que resulta cognitivamente menos válido y que puede corresponder a un conocimiento meramente disciplinar. Esto deriva, al igual que el grupo de profesores de biología, en una formación científica inadecuada para el desarrollo de la competencia transversal. El resultado pone de manifiesto una presumible influencia de las habilidades desarrolladas y predominantes durante la formación de cada profesional que posteriormente enseña ciencias. Esta conjetura reside en que los profesionales que tienen una formación técnico-científica se destacan en la capacidad para la resolución de problemas descontextualizados, en el que prevalece el conocimiento de las ciencias y carecen de conocimientos sobre la NdcyT.

\section{CONCLUSIÓN}

Del contraste y validez de los resultados empíricos es posible afirmar que los docentes participantes con formación psicopedagógico-didáctica, y particularmente los profesores de fisicoquímica, poseen un conocimiento didáctico más específico y apropiado sobre el contenido disciplinar. Esa realidad evidencia las diferencias respecto de los profesores de biología y el resto de los profesionales con formación técnico-disciplinar.

Este contraste de resultados en función del perfil de profesionales y de la especificidad disciplinar que enseñan abre la necesidad de profundizar en nuevas indagaciones para identificar habilidades pedagógico-didácticas específicas sobre el contenido. Dado que el grupo de educadores que enseña fisicoquímica alcanzó cambios significativos en tres de las cinco habilidades, respecto del grupo de educadores que enseña biología, la diferencia en los resultados permite inferir que el segundo grupo de profesores y los de formación técnico-disciplinar requieren ciertamente prestar mayor atención en analizar y caracterizar sus habilidades de pensamiento crítico para construir su CDC. Efectivamente, esto les permitiría enfrentar situaciones didácticas específicas en determinados contenidos para la enseñanza de la ciencia y la tecnología desde un conocimiento metacientífico más cabal.

Con seguridad y en relación a este último señalamiento en la cuestión, los resultados del presente informe estimulan la necesidad de correlacionar el conocimiento sobre las ideas metacientíficas con las habilidades del PC, pero su mero tratamiento excede el propósito de este documento.

Sin duda, el concepto de competencia transversal muestra un carácter polisémico y multidimensional, lo cual deriva en muchos y variados matices para precisar las cualidades de los profesionales científicamente alfabetizados en todos los campos disciplinares. Se advierte que el potencial del tipo de estudio empleado 
CARACTERIZACIÓN DE LAS HABILIDADES DEL PENSAMIENTO CRÍTICO PARA EL DESARROLLO

DEL CONOCIMIENTO DIDÁCTICO DEL CONTENIDO EN PROFESORES DE CIENCIAS NATURALES

permite identificar y cuantificar las diferentes habilidades por contraste de hipótesis. Además, su caracterización como habilidades transversales viabiliza la evaluación de tipologías de procesamiento cognitivo de manera integral, desde la perspectiva del análisis cuantitativo y cualitativo. Esto, a su vez, permite encontrar formas de analizar y fortalecer el trabajo docente para el desarrollo de la competencia transversal y específica de manera menos subjetiva y más rigurosa.

En definitiva, conocer y caracterizar los patrones de razonamiento y los recursos cognitivos que los docentes utilizan a partir de las habilidades del pensamiento crítico permitiría avanzar en el desarrollo del conocimiento didáctico del contenido del profesorado. Convincentemente, es deseable que la formación del profesorado en ciencias naturales oriente aquellas competencias que deben formar parte del conjunto del conocimiento didáctico crítico que se desee promover en los futuros docentes.

Agradecimientos: proyecto de investigación La Enseñanza de la Naturaleza de la Ciencia y la Tecnología (NdCyT) y el desarrollo del Pensamiento Crítico (PC), EDU2015-64642-R (MINECO/FEDER); FI 04/1-208 (UNCO).

\section{REFERENCIAS BIBLIOGRÁFICAS}

Abd-el-Khalick, F. y Akerson, V. (2009). The Influence of Metacognitive Training on Preservice Elementary Teachers. Conceptions of Nature of Science. International Journal of Science Education, 31, 2161-2184.

Acevedo-Díaz, J. A. y García-Carmona, A. (2016). "Algo antiguo, algo nuevo, algo prestado». Tendencias sobre la naturaleza de la ciencia en la educación científica. Revista Eureka sobre Enseñanza y Divulgación de las Ciencias, 13 (1), 3-19. Recuperado de http://hdl. handle.net/10498/18010.

Álvarez-Suárez, P.; Cepero-Espinosa, S.; Arce, C. y Perales-Palacios, F. J. (2013). Posibilidades del escalamiento subjetivo como técnica de análisis del pensamiento espontáneo del profesorado de ciencias en formación. Profesorado, Revista de Currículum y Formación de Profesorado, 17 (2), 347-368.

Blanco, L. J.; Mellado, V. y Ruiz, C. (1995). Conocimiento didáctico del contenido en ciencias experimentales y matemáticas y formación de profesores. Revista de Educación, 307, 427-446.

Coronado, M. (2013). Competencias docentes: ampliación, enriquecimiento y consolidación de la práctica profesional. Buenos Aires: Noveduc.

Dewey, J. (1998). Cómo pensamos. Nueva exposición de la relación entre el pensamiento reflexivo y proceso educativo. Barcelona: Paidós.

Ennis, R. H. (1993, verano). Critical thinking assessment [Evaluación del pensamiento crítico]. Theory Intro Practice, 32 (3), 179-186. Recuperado de http://www3.qcc.cuny.edu/WikiFiles/file/Ennis\%20Critical\%20Thinking\%20Assessment.pdf.

Ennis, R. H. (1996). Critical thinking dispositions: their nature and assessibility [Disposiciones del pensamiento crítico: Su naturaleza y accesibilidad]. Informal Logic, 18 (2-3), 165182. Recuperado de http://ojs.uwindsor.ca/ojs/leddy/index.php/informal_logic/article/ viewFile/2378/1820. 
MARCELO AUGUSTO SALICA

CARACTERIZACIÓN DE LAS HABILIDADES DEL PENSAMIENTO CRÍTICO PARA EL DESARROLLO

DEL CONOCIMIENTO DIDÁCTICO DEL CONTENIDO EN PROFESORES DE CIENCIAS NATURALES

Facione, P. (1990). The California Critical Thinking Skills Test (CCTST): Forms A and B; and the CCTST test manual. Millbrae, CA: The California Academic Press.

Farré, A. S. y Lorenzo, M. G. (2009). Conocimiento Pedagógico del Contenido: Una definición química. Educación en la Química, 15 (2), 103-113.

Garritz, A. (2010). La enseñanza de la ciencia en una sociedad con incertidumbre y cambios acelerados. Enseñanza de las Ciencias, 28 (3), 315-326.

Garritz, A. (2013). PCK for dummies. Educación Química, 24 (e2), 462-465.

Garritz, A.; Daza Rosales, S. F. y Lorenzo, M. G. (2014). Conocimiento didáctico del contenido: una perspectiva iberoamericana. Alemania: Académica Española.

Garritz, A. y Trinidad-Velasco, R. (2004). El conocimiento pedagógico del contenido. Educación Química, 15 (2), 98-102.

Halpern, D. (1998). Teaching critical thinking for transfer across domains [La enseñanza del pensamiento crítico para la transferencia a través de dominios]. American Psychologist Associations, 53 (4), 449-455. Recuperado de http://dx.doi.org/10.1037/0003066X.53.4.449.

Halpern, D. F. (2006). Is intelligence critical thinking? Why we need a new construct definition for intelligence. En P. Kyllonen, I. Stankov y R. D. Roberts (Eds.). Extending intelligence: Enhancement and new constructs. Mahwah, NJ: Erlbaum Associates.

Hernández, S. R.; Fernández, C. C. y Baptista, L. P. (2006). Metodología de la investigación. México: McGraw-Hill.

Imbernón, F. (2010). La formación inicial y permanente del profesorado de secundaria. Hacia un nuevo concepto de formación del profesorado. Alambique: Didáctica de las Ciencias Experimentales, 65, 65-72.

Kuhn, D. (marzo, 1999). A developmental model of critical thinking [Un modelo de desarrollo del pensamiento crítico]. Educational Researcher, 28 (2), 16-46. doi: http://dx.doi. org/10.3102/0013189X028002016.

Loughran, J.; Berry, A. y Mulhall, P. (Eds.) (2012). Understanding and Developing Science Teachers' Pedagogical Content Knowledge. Rotterdam: Sense Publishers.

Lipman, M. (septiembre, 1988). Critical thinking -what can it be? [Pensamiento crítico - ¿lo que puede ser?]. Educational Leadership.

López, G. (enero-diciembre, 2012). Pensamiento crítico en el aula. Docencia e Investigación, 37 (22), 41-60. Recuperado de http://educacion.to.uclm.es/pdf/revistaDI/3_22_2012. pdf.

Martín-Barbero, J. (2003). Competencias transversales del sujeto que aprende. Sinéctica, Revista Electrónica de Educación, (22), 30-36.

Mellado, V. (1999). La investigación sobre la formación del profesorado de Ciencias Experimentales. En C. Martínez y S. García (Eds.). La Didáctica de las Ciencias. Tendencias actuales. XVIII Encuentros de Didáctica de las Ciencias Experimentales (pp. 45-76). La Coruña: Servicio de Publicaciones de la Universidad de La Coruña.

Molina-Patlán, C.; Morales-Martínez, G. P. y Valenzuela-González, J. R. (enero-abril, 2016). Competencia transversal, pensamiento crítico: Su caracterización en estudiantes de una secundaria de México. Revista Electrónica Educare, 20 (1), 1-26. Recuperado de http:// dx.doi.org/10.15359/ree.20-1.11

Nieto, A. M. y Saiz, C. (2011). Skills and dispositions of critical thinking: Are they sufficient? [Habilidades y disposiciones sobre el pensamiento crítico: ¿Son suficientes?]. Anales de Psicología, 27 (1), 202-209. Recuperado de http://revistas.um.es/analesps/article/ view/113641/107631. 
CARACTERIZACIÓN DE LAS HABILIDADES DEL PENSAMIENTO CRÍTICO PARA EL DESARROLLO DEL CONOCIMIENTO DIDÁCTICO DEL CONTENIDO EN PROFESORES DE CIENCIAS NATURALES

Nieto, A. M., Saiz, C. y Orgaz, B. (2001). Análisis de las propiedades psicométricas de la versión española del HCTAEs-Test de Halpern para la evaluación del pensamiento crítico mediante situaciones cotidianas. Revista Electrónica de Metodología Aplicada, 14 (1), 1-15. Recuperado de https://www.unioviedo.es/reunido/index.php/Rema/article/ view/9786/9525.

Organización para la Cooperación y el Desarrollo Económico (OCDE, 2005). La definición y selección de competencias clave (Resumen ejecutivo). Recuperado de http://www. deseco.admin.ch/bfs/deseco/en/index/03/02.parsys.78532.downloadList.94248.DownloadFile.tmp/2005.dscexecutivesummary.sp.pdf.

Organización para la Cooperación y el Desarrollo Económico (OCDE, 2010). Habilidades y competencias del siglo XXI para los aprendices del nuevo milenio en los países de la OCDE. París: Instituto de Tecnologías Educativas. Recuperado de http://recursostic. educacion.es/blogs/europa/media/blogs/europa/informes/Habilidades_y_competencias_siglo21_OCDE.pdf.

Padilla, K.; Ponce de León, A. M.; Rembado, F. M. y Garritz, A. (2008). Undergraduate, Professor's Pedagogical Content Knowledge: the case of amount of substance. International Journal of Science Education, 30 (10), 1389-1404.

Paul, R. y Elder, L. (2003). La mini-guía para el pensamiento crítico, conceptos y herramientas. California: Fundación para el Pensamiento Crítico. Recuperado de https://www. criticalthinking.org/resources/PDF/SP-ConceptsandTools.pdf.

Pedrinaci, E. (Coord.); Caamaño, A.; Cañal, P. y De Pro, A. (2012). El desarrollo de la competencia científica: 11 ideas clave. Barcelona: Editorial Graó.

Mora, W. M. y Parga, D. L. (2014). Aportes al CDC desde el pensamiento complejo. En A. Garritz (Ed.), Lorenzo, M. G., Daza Rosales, Silvio F. (2014). Conocimiento didáctico del contenido: una perspectiva iberoamericana (p. 115). Alemania: Académica Española.

Pozo, J. I. y Carretero, M. (1987). Del pensamiento formal a las concepciones espontáneas: ¿Qué cambia en la enseñanza de la ciencia? Infancia y Aprendizaje, 35-52. Recuperado de https://dialnet.unirioja.es/descarga/articulo/662329.pdf.

Shulman, L. S. (1987). Knowledge and teaching: Foundations of the new reform. Harvard Educational Review, 57 (1), 1-22.

UnesCo (2005). Hacia las sociedades del conocimiento. París: Autor. Recuperado de http:// unesdoc.unesco.org/images/0014/001419/141908s.pdf.

Vázquez, A. A. y Rodríguez, C. A. M. (septiembre, 2013). Secuencia de enseñanza aprendizaje sobre la naturaleza de la ciencia y la tecnología para la formación del profesorado de ciencias. IX Congreso Internacional sobre Investigación en Didáctica de las Ciencias. Girona.

Verdugo-Perona, J. J.; Solaz-Portolés, J. J. y Sanjosé-López, V. (2017). El conocimiento didáctico del contenido en ciencias: estado de la cuestión. Cuadernos de Pesquisa, 47 (164), 566-611. Recuperado de http://dx.doi.org/10.1590/198053143915. 